\section{LAW OF THE MOVING-IRON ELECTRICAL INSTRUMENT}

Tre HE law of the moving-iron instrument has been given in the form, torque equals $\frac{1}{2} I^{2} d L / d \theta$, where $I$ is the current in the coil and $d I J / d \theta$ the rate of change of inductance with angular deflexion. This law assumes the current remains constant for a small change in deflexion, but since a change in inductance is produced by a change in current, the assumption does not seem justifiable. The law therefore takes a mure complicated form, and in a paper on the subject (J. Inst. Elec. Eng., 90, Pt. II, No. 13, February 1943) Dr. G. F. Tagg describes experiments which show that the simple law does not hold for a simple electromagnetic system, and it is considered that the law as enunciated in most text-books is incorrect.

Moving-iron instruments do not generally lend themselves to any simple theoretical treatment, and the very nature of the instrument makes any calculation of the current-torque relation very difficult, if not impossible. It is stated in the proof of text-book equations governing the law that the energy of the coil is $\frac{1}{2} L I^{2}$ and that it is a change in this energy which produces the pull. The pull is exerted on the iron, and there is no justification for assuming that the whole of the magnetic energy is used to produce it. It is the magnetic potential of the iron which matters, and this is not necessarily the same as that of the coil. Again, the statement is made "if the current remains constant"; but this cannot apply as a change in current produces a change in deflexion, and it is not possible to have an effect and to assume that the cause is zero.

It is suggested that the true equations are, in the case of an angular deflection $\theta$, the torque

$$
T=\frac{d\left(\frac{1}{2} \eta L I^{2}\right)}{d \theta}=\frac{1}{2} \eta I^{2} \frac{d L}{d \theta}+\frac{1}{2} I^{2} L \frac{d \eta}{d \theta}+L I \eta \frac{d I}{d \theta},
$$

and that for a linear deflexion $x$, the pull

$$
F=\frac{d\left(\frac{1}{2} \eta L I^{2}\right)}{d x}=\frac{1}{2} \eta I^{2} \frac{d L}{d x}+\frac{1}{2} I^{2} L \frac{d \eta}{d x}+L I \eta \frac{d I}{d x},
$$

in which $\eta$ represents the magnetic efficiency. The available energy to produce torque or force is $\frac{1}{2} \eta L I^{2}$; $\eta, L$ and $I$ can all be regarded as functions of $x$.

The true expression for the torque or pull is thus more complicated than the simple ones usually put forward and involves a quantity $\eta$, the 'magnetic efficiency', the value of which it is practically impossible to predetermine. It is therefore a matter of extreme difficulty to forecast the behaviour of any particular instrument, and experiment appears to be the only solution.

Dr. C. V. Drysdale, dealing with the "Torque of Soft-Iron Electrical Instruments" ( $J$. Inst. Elec. Eng., 90, Pt. II, No. 13, February 1943) shows that the inductance formulæ $F=\frac{1}{2} I^{2} \delta L / \delta x$ and $T=\frac{1}{2} I^{2} \delta L / \delta \theta$ are correct for iron-cored instruments if the magnetization of the iron does not exceed the maximum value permissible for accurate indication. For higher magnetizations the force or torque is higher than the value given by the formulæ, and becomes twice as great when the iron approaches saturation.

The inductance method of calculating the torque of electromagnetic instruments is so valuable, both for design purposes and for the experimental shaping of the moving iron without the laborious process of dismantling, rebalancing and reassembling the movement for each modification, that it has now been fairly widely adopted. The experiments carried out by Dr. Tagg referred to above have led him to claim, however, that the method is based on unjustifiable assumptions and has no experimental confirmation; it is true that it is applicable only when the magnetiza. tion of the iron does not exceed a certain value; but this limitation does not affect its utility for the development of accurate instruments.

In view of the above claim and of the importance and limitation of the method, it is desirable that its theory should be completed, established and extended. It is based on the principle of the conservation of energy, and is so closely analogous for electrostatic and electromagnetic instruments that it is advantageous to consider them together; this is done in the paper referred to. It is shown that the formulie already mentioned are applicable to softiron instruments if the magnetic flux on the iron is proportional to the current. For electrostatic instruments the capacitance is independent of the potential difference, so that the formulæ $f=\frac{1}{2} V^{2} \delta C / \delta x$ and $T^{\prime}==\frac{1}{2} V^{2} \delta C / \delta \theta$ are always valid. The paper also disuusses the effect of variable permeability of the iron circuit of electromagnetic instruments.

\section{PRESERVATION OF TIMBER-IN RHODESIA}

$\mathrm{R}$ H. FINLAY, forest officer, Department of Agriculture, Rhodesia, has discussed the preservation of timber in that country (Proc. Rhodesia Sci. Assoc., 39, 103; 1942). After dealing with some of the earliest methods of utilizing durable timbers, Mr. Finlay comes to the period when, with the greater utilization by man of varying timbers from the forests and the consequent necessity for extending the period of utility of the softer woods, attempts were made to introduce preservative treatment. Mercuric chloride and copper sulphate had already been recommended in the eighteenth century, but methods of treatment with these were not patented until the 1830's. In 1832 Kyan patented a method based on mercuric chloride or corrosive sublimate, which came to be known as 'kyanizing'. Sir William Burnett then made use of zinc chloride, and the method was patented in 1838. Originally this consisted in immersing the timber in open tanks of zine chloride; the method is still used. Later it was modified to a pressure process known as 'burnettizing'. Creosote, even more familiar to-day on the countryside in England, was also experimented with at this time, and in $1838 \mathrm{John}$ Bethell was granted a patent to inject creosote under pressure. The modern Bethell or full-cell process is the direct outcome of this process. As is well known, attention became more and more fixed on the use of creosotes and mixtures containing it, and they hold high rank to-day.

In recent years the market has had a sequence of proprietary preservatives, but for the most part they cannot be said to be truly established as commercial timber preservatives. Moreover, as Mr. Finlay goes on to show, different climatic conditions affect very considerably the methods which it may be possible to introduce. For example, protection against the termite may vary in dry hot as against moist hot localities. Further, there are innumerable borer and fungus attacks to be considered, including the marine borer. An interesting example of present knowledge on the subject is connected with a fungus growth 